Article

\title{
Pyrosequencing Reveals the Predominance of Pseudomonadaceae in Gut Microbiome of a Gall Midge
}

\author{
Raman Bansal $^{1, \dagger}$, Scot H. Hulbert ${ }^{2}$, John C. Reese ${ }^{1}$, Robert J. Whitworth ${ }^{1}$, Jeffrey J. Stuart ${ }^{3}$ \\ and Ming-Shun Chen ${ }^{1,4, *}$
}

1 Department of Entomology, Kansas State University, Manhattan, KS 66506, USA;

E-Mails: bansal.67@osu.edu (R.B.); jreese@ksu.edu (J.C.R.); jwhitwor@ksu.edu (R.J.W.)

2 Department of Plant Pathology, Washington State University, Pullman, WA 99164, USA;

E-Mail: scot_hulbert@wsu.edu

3 Department of Entomology, Purdue University, West Lafayette, IN 47907, USA;

E-Mail: stuartjj@purdue.edu

4 USDA-ARS, Hard Winter Wheat Genetics Research Unit, 4008 Throckmorton Hall, Kansas State University, Manhattan, KS 66506, USA

$\uparrow$ Current address: Department of Entomology, Ohio Agricultural Research and Development Center, The Ohio State University, Wooster, OH 44691, USA.

* Author to whom correspondence should be addressed; E-Mail: mchen@ksu.edu; Tel.: +1-785-532-4719; Fax: +1-785-532-6232.

Received: 1 April 2014; in revised form: 13 May 2014 / Accepted: 3 June 2014 /

Published: 11 June 2014

\begin{abstract}
Gut microbes are known to play various roles in insects such as digestion of inaccessible nutrients, synthesis of deficient amino acids, and interaction with ecological environments, including host plants. Here, we analyzed the gut microbiome in Hessian fly, a serious pest of wheat. A total of 3,654 high quality sequences of the V3 hypervariable region of the $16 \mathrm{~S}$ rRNA gene were obtained through 454-pyrosequencing. From these sequences, 311 operational taxonomic units (OTUs) were obtained at the $\geq 97 \%$ similarity cutoff. In the gut of 1 st instar, otu01, a member of Pseudomonas, was predominant, representing $90.2 \%$ of total sequences. otu13, an unidentified genus in the Pseudomonadaceae family, represented $1.9 \%$ of total sequences. The remaining OTUs were each less than $1 \%$. In the gut of the 2 nd instar, otu01 and otu 13 decreased to $85.5 \%$ and $1.5 \%$, respectively. otu04, a member of Buttiauxella, represented $9.7 \%$ of total sequences. The remaining OTUs were each less than $1 \%$. In the gut of the 3 rd instar, otu01
\end{abstract}


and otu13 further decreased to $29.0 \%$ and $0 \%$, respectively. otu06, otu08, and otu16, also three members of the Pseudomonadaceae family were $13.2 \%, 8.6 \%$, and $2.3 \%$, respectively. In addition, otu04 and otu14, two members of the Enterobacteriaceae family, were $4.7 \%$ and $2.5 \%$; otu 18 and otu 20 , two members of the Xanthomonadaceae family, were $1.3 \%$ and $1.2 \%$, respectively; otu12, a member of Achromobacter, was $4.2 \%$; otu19, a member of Undibacterium, was 1.4\%; and otu9, otu10, and otu15, members of various families, were $6.1 \%, 6.3 \%$, and $1.9 \%$, respectively. The investigation into dynamics of Pseudomonas, the most abundant genera, revealed that its population level was at peak in freshly hatched or 1 day larvae as well as in later developmental stages, thus suggesting a prominent role for this bacterium in Hessian fly development and in its interaction with host plants. This study is the first comprehensive survey on bacteria associated with the gut of a gall midge, and provides a foundation for future studies to elucidate the roles of gut microbes in Hessian fly virulence and biology.

Keywords: Hessian fly; Mayetiola destructor; gut; microbe; symbiosis

\section{Introduction}

The gut microbiome consists of microorganisms that live in the digestive tracts of animals, and is an important component of the gut of an organism [1]. There have been numerous studies on the characterization of gut microbiome in insects such as pea aphid, fruit fly, honey bee etc. which were reviewed recently [2]. The composition of gut microbiome varies greatly from species to species. Even within the same species, variation in gut microbiome occurs among different individuals, which causes phenotypic differences among these individuals [3]. Diverse microbiome is expected in the gut of insects since different species live in very different ecological environments, and utilize a wide range of food sources from plant tissues to human blood [4]. The insect gut microbes performs a wide range of functions useful to the host, such as synthesizing vitamins and essential amino acids, preventing growth of harmful pathogens, and utilizing energy substrates that cannot be used directly by the host itself [5]. Additionally, insect gut microbes are involved in detoxification of plant secondary metabolites and modification of host plant recognition [6]. Thus, owing to these diverse and vital roles, gut microbes are believed to be key contributors in evolutionary adaptations and ecological success of insects [2].

Gall midges consist of one of the largest and most diversified families in Insecta [7]. Most plantfeeding gall midges induce the formation of various types of galls, and many of them are economically important pests in agriculture [8]. So far, no gut microbiota has been systematically characterized from a gall midge. Such studies would provide useful information for a more comprehensive understanding of the biology and ecology of these insects. Hessian fly, Mayetiola destructor, is a gall midge and one of the most destructive pests of wheat $[9,10]$. After hatching, neonatal larvae migrate along the leaf and between leafsheaths all the way down to the base of a plant [7]. A first instar larva establishes a permanent feeding site between leaf-sheaths and induces the formation of nutritive cells at the feeding site [11]. The larva stays there and sucks up cell content of nutritive cells until the insect becomes a pupa. The Hessian fly 
larval gut is one of the most important interfaces for the interaction between the insect and its host plants. There is evidence suggesting that the Hessian fly larval gut contains diverse bacteria such as Enterobacter, Stenotrophomonas, Pseudomonas, and Bacillus etc. [12-15]. Further characterization of the gut microbiota of Hessian fly larvae will provide useful information for research on the ecological and molecular interactions among the Hessian fly, its host plants, and its symbiotic microorganisms.

Lately, high throughput technologies focusing on bacterial 16S rRNA gene have been applied to analyze insect gut microbiomes [16-18]. The 16S rRNA gene is generally highly conserved, but between the highly conserved regions, hypervariable regions exist [19]. A total of nine hypervariable regions, named V1 to V9, are present in the 16S rRNA gene. This genic structure makes the 16S rRNA gene useful for microbial identification and phylogenetic analysis [20]. PCR amplification and subsequent sequence analysis of the $16 \mathrm{~S}$ rRNA gene makes it possible to identify microbes without culturing them. In general, the sequence information of either V3 or V6 variable regions alone provides sufficient data for phylogenetic analysis to the genus level [21]. In this study, we conducted pyrosequencing of the V3 region (corresponding to residues 341 to 534 of the Escherichia coli 16S rRNA gene) of DNA samples extracted from the guts of different instars of Hessian fly larvae and amplified directly using PCR. Further, we determined the population dynamics of Pseudomonas, the most abundant genera, in different developmental stages of Hessian fly.

\section{Results and Discussion}

To assess the diversity of microbes and their relative richness in the gut of different instars, a total of 6062 reads were obtained for the V3 region of the 16S rRNA gene through 454-pyrosequencing. After removing low-quality reads, 5522 reads were retained for further analysis. Taxonomic classification using ARB-Silva database placed 612 of the reads to the "cyanobacteria-chloroplast" group. The subsequent blastn search using wheat chloroplast genome (GenBank: KC912694.1) as reference confirmed that these reads were derived from wheat chloroplast 16S DNA in the Hessian fly larval gut obtained during feeding. In addition, 494 reads were placed to the Magnoliophyta group (Supplementary Table S1), which were likely derived from wheat 18S DNA present in Hessian fly larval gut since Triticum aestivum is a member of the Magnoliophyta flowering plants. This was confirmed through blastn search as these reads showed $99 \%-100 \%$ identity to wheat mitochondrion genome (GenBank: GU985444.1). Furthermore, 762 reads were assigned to the Insecta group (Supplementary Table S2). The subsequent blastn search using hessian fly as reference revealed that these reads were derived from Hessian fly 18S DNA (GenBank: KC177284.1). Apparently, the 341F-529R primer pair amplified both wheat and Hessian fly ribosomal genes due to their degenerate nature.

The remaining 3654 reads was subjected to operational taxonomic unit (OTU) clustering at the $\geq 97 \%$ similarity cutoff (Table 1 ). The OTU clustering and subsequent rarefaction analysis allowed the comparison of species-richness (number of OTUs) between different larval instars and the determination of adequacy of sequencing output for each stage. The slopes (with respect to $x$-axis) for the Hfg1 and Hfg3 curves were steeper than that from Hfg2 (Figure 1), indicating that for the same number of sequence reads, the 1 st and 3rd instar larval guts have much higher richness than the 2nd instar. The rarefaction curve for Hfg2 appeared to reach the plateau/stationary phase (along $x$-axis) but the curves for Hfg1 and Hfg3 did not show proclivity towards $x$-axis. However, the values for "Good's 
coverage" for sequencing data in all samples including $\mathrm{Hfg} 1$ and $\mathrm{Hfg} 3$ (at $\geq 97 \%$ similarity cutoff) ranged between $88.7 \%-97.7 \%$ (Table 1). Taken together, these results suggest that although more species would be expected in the 1st and 3rd instar larval guts with additional sequencing, most bacterial diversity from all samples has already been captured.

Table 1. Non-parametric estimates of coverage and species richness.

\begin{tabular}{|c|c|c|c|c|}
\hline$\downarrow_{\vee \% \text { Similarity }}{ }^{a}$ & Sample ${ }^{b}$ & Hfg1 & Hfg2 & Hfg3 \\
\hline \multirow{3}{*}{$\geq 99$} & Coverage $(\%)$ & 92.0 & 94.6 & 81.8 \\
\hline & Ace $^{c}$ & $1380.9(1156.2,1657.1)$ & $449.2(331.8,645.3)$ & $3004.3(2627.2,3442.3)$ \\
\hline & Chao $^{c}$ & $433.1(331.8,599.9)$ & $410.1(304.9,590.2)$ & $1284.1(917.1,1867.4)$ \\
\hline \multirow{3}{*}{$\geq 97$} & Coverage $(\%)$ & 95.4 & 97.7 & 87.8 \\
\hline & Ace & $440.0(323.1,621.1)$ & $201.9(132.8,347.2)$ & $1411.7(1196.9,1673.2)$ \\
\hline & Chao & $207.3(162.9,289.0)$ & $187.6(122.8,332.0)$ & $767.8(537.6,1159.2)$ \\
\hline \multirow{3}{*}{$\geq 95$} & Coverage $(\%)$ & 96.9 & 98.5 & 90.1 \\
\hline & Ace & $182.8(126.7,299.7)$ & $128.7(82.9,234.8)$ & $1132.9(958.5,1346.1)$ \\
\hline & Chao & $153.4(112.0,241.2)$ & $103.1(70.0,186.2)$ & $521.7(375.7,774.5)$ \\
\hline \multirow{3}{*}{$\geq 90$} & Coverage $(\%)$ & 98.5 & 99.2 & 93.8 \\
\hline & Ace & $118.7(88.6,170.0)$ & $66.1(41.6,138.4)$ & $463.8(375.6,582.9)$ \\
\hline & Chao & $73.6(56.1,121.4)$ & $80.0(44.0,202.5)$ & $281.3(206.6,423.0)$ \\
\hline
\end{tabular}

${ }^{\mathrm{a}}$ Pyrosequencing reads with a given level of sequence similarity were grouped together; ${ }^{\mathrm{b}} \mathrm{Hfg} 1$ : first instar larvae gut (1-3 days old); Hfg2: second instar larvae gut (6-8 days old); Hfg3: third instar larvae gut (13-15 days old); ${ }^{c}$ Ace and Chao are non-parametric measures of species richness. Lower and higher limits (at 95\% confidence interval) for these estimates are indicated in parentheses.

Figure 1. Rarefaction analysis based on resampling without replacement approach.

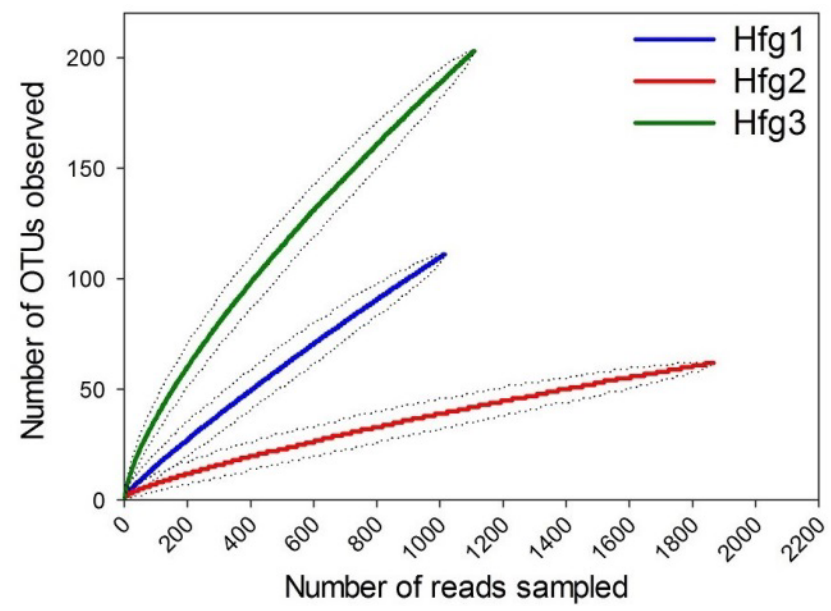

Rarefaction curves are shown for operational taxonomic units (OTUs) at $\geq 97 \%$ similarity cutoff for all three samples Hfg1, Hfg2 and Hfg3. Dotted lines represent the upper and lower limits at 95\% confidence level. Hfg1: Frist instar larval gut (1-3 days old); Hfg2: Second instar larval gut (6-8 days old); and Hfg3: Third instar larval gut (13-15 days old). The analysis was performed using Mothur [22].

At $\geq 97 \%$ similarity cutoff, the 3654 reads from the three samples were binned into 311 OTUs. Among these OTUs, 229 contained only single reads. These single-read OTUs could either be derived from Hessian fly symbiotic bacteria with very low abundance or due to low amplification efficiency 
of the 16S DNA from specific bacteria. Alternatively, these single-read OTUs could be due to contamination or sequencing errors during the amplification and sequencing process. The remaining 82 multi-read OTUs (representing 3425 reads, or $93.7 \%$ of the total bacterial reads) were subjected to further analysis since they were likely to represent important symbiotic bacteria in the Hessian fly larval gut. As shown in Figure 2, the 82 OTUs were derived from all three samples, but the Hfg3-sample contributed most of them. Fifty-five of the 82 multi-read OTUs, representing $96.53 \%$ of the reads contained in these OTUs, were assigned to phylum Proteobacteria, and remaining 27 OTUs were assigned to either Actinobacteria (8 OTUs), Bacteroidetes (3 OTUs), Acidobacteria (2 OTUs), or unclassified (14 OTUs). Among the proteobacterial OTUs, 25 were assigned to Gammaproteobacteria (representing $91.89 \%$ of the Proteobacteria reads), 15 to Alphaproteobacteria (5.08\% of the Proteobacteria reads), and 14 Betaproteobacteria (2.96\% of the Proteobacteria reads), and one unclassified. The Gammaproteobacteria and Actinobacteria reads were found in gut of all three instars whereas Alphaproteobacteria reads were found only in first and third instar stages. However, the Betaproteobacteria and Flavobacteria reads were detected only in the gut of third instar larvae.

Figure 2. Bacterial OTUs identified from the gut of Hessian fly larvae.

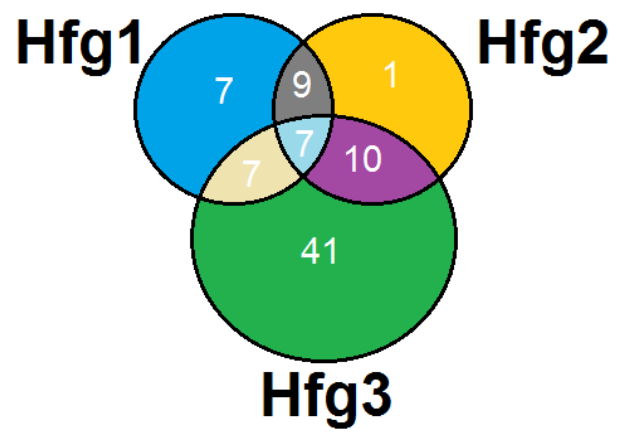

Venn diagram showing the number of OTUs (at $\geq 97 \%$ similarity cutoff) that are either unique to or shared between the gut samples. Hfg1: Frist instar larval gut (1-3 days old); Hfg2: Second instar larval gut (6-8 days old); and Hfg3: Third instar larval gut (13-15 days old).

The phylogenetic relationship and distribution of sequence reads of the 82 OTUs are shown in Supplemental Figure S1. Since the top 20 OTUs represent $94.3 \%$ the bacteria reads, we focused on these OTUs for comparative analysis of bacteria abundance and diversity in different Hessian fly larval instars. Phylogenetic grouping resulted in four major clusters (Figure 3). Cluster I, the largest group with seven OTUs and 2713 (or 74.2\%) reads, belongs to Pseudomonadaceae. Four of these OTUs, namely otu01, otu06, otu16, and otu24 were identified as Pseudomonas, whereas the other three could not be identified to the genus level. otu01 was the most abundant in all three samples. Cluster II, containing four OTUs and 272 (or 7.5\%) reads belongs to Enterobacteriaceae. Amongst these OTUs, otu04 was identified as Buttiauxella, whereas the other three (otu14, otu25, otu26) could not be identified to the genus level at present. Cluster III consists of two OTUs, otu18 (12 reads, Stenotrophomonas) and otu20 (15 reads, unclassified). Cluster IV contains four OTUs and 153 reads, which have diverse bacterial lineages. In cluster IV, otu23 was identified as Chrysobacterium (Bacteroidetes), otu15 as Propionibacterium (Actinobacteria), and otu09 and otu10 were identified as Rhizobiales (Alphaproteobacteria). Three of the 20 abundant OTUs, otu21 (Acinetobacter, Moraxellaceae), 
otu12 (Achromobacter, Alcaligenaceae), and otu19 (Undibacterium, Oxalobacteriaceae), could not be placed into any of the major clusters.

Figure 3. Relative abundance of the top 20 OTUs in different Hessian fly larval instars and their phylogenic relationship.
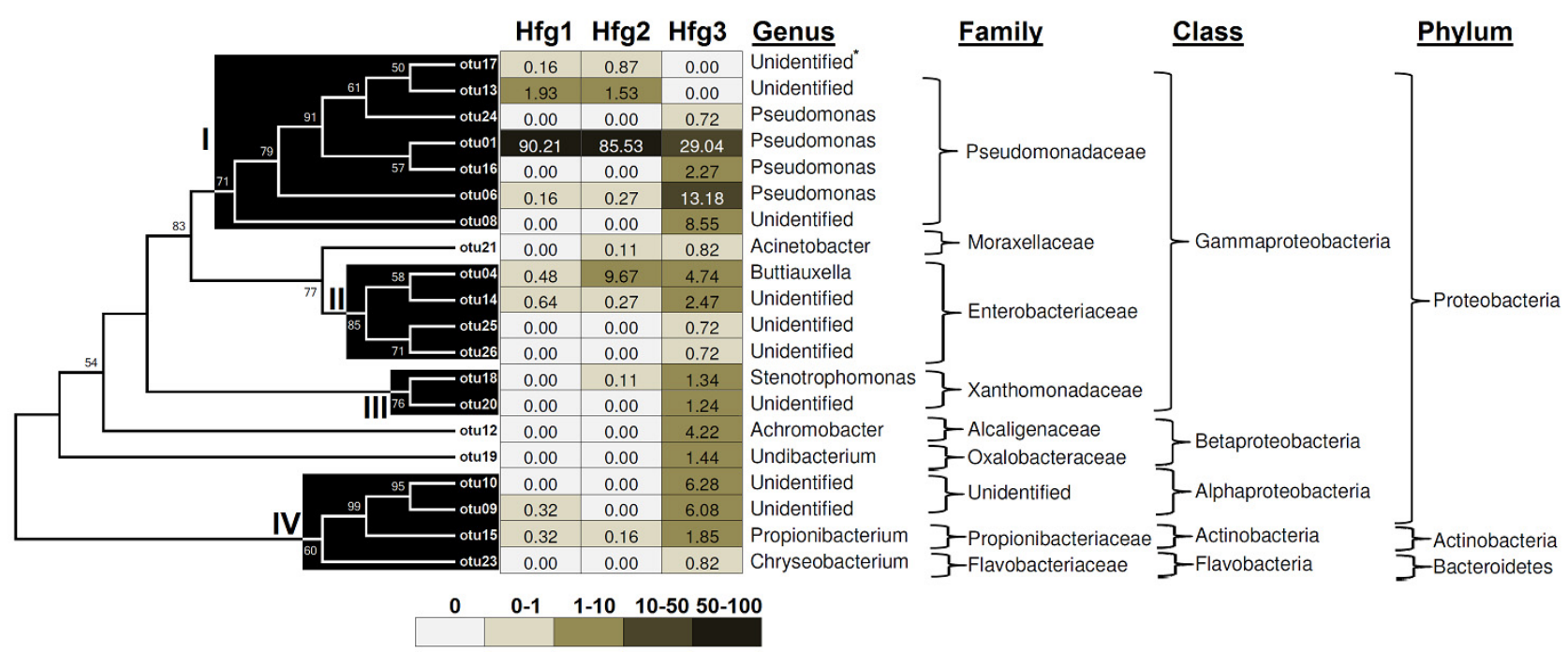

Relative abundance (\%) of an OTU in a sample was calculated by dividing the number of the corresponding OTU reads with the total bacterial reads. Levels of relative abundances are illustrated by a color scale standing for $0 \%, 0 \%-1 \%, 1 \%-10 \%, 10 \%-50 \%$, and $50 \%-100 \%$, respectively. Representative sequence for each of top 20 OTUs was used to deduce the phylogenetic inference through Maximum Likelihood method based on Tamura-Nei model [23] in MEGA5.05 [24]. Percentages of replicate trees in which OTU sequences clustered together in the bootstrap test (1000 replicates) are shown (only above 50\%) next to the branches in unrooted phylogenetic tree [25]. Taxonomic assignments at genus, class, family, and phylum levels are indicated [*otu17 was not classifiable beyond domain (bacteria) level]. Hfg1: First instar larval gut (1-3 days old); Hfg2: Second instar larval gut (6-8 days old); and Hfg3: Third instar larval gut (13-15 days old).

The composition of gut bacteria varied in different larval instars and diversity increased in later larval stages. In the gut of the 1 st instar, $90.2 \%$ sequences belong to a single OTU, otu01. The second most abundant OTU, otu13 (also a member of Pseudomonas), contained 1.9\% sequences; and the remaining OTUs each contained less than $1 \%$ sequences. The composition of gut bacteria changed significantly in the 2nd instar. otu01 was still the most abundant in the gut of 2 nd instar larvae, but decreased to $85.5 \%$. otu 13 also decreased to $1.5 \%$. otu 04 , on the other hand, increased dramatically from less than $1 \%$ in 1 st instar to $9.7 \%$ in 2 nd instar. otu04 belongs to Buttiauxella in the Enterobacteriaceae family. The gut bacteria in 3rd instar larvae were the most diversified. Again, otu01 was still the most abundant in the gut of 3rd instar, but decreased to only $29.0 \%$. Otu06 (also Pseudomonas) became the second most abundant unit, representing $13.2 \%$ of the sequences. In addition, 11 more OTUs were with relative abundance more than 1\%, including otu04 (Buttiauxella, 4.7\%), otu08 (a member of the Pseudomonadaceae, 8.6\%) otu09 (a member of Alphaproteobacteria, 6.1\%), otu10 (a member of Alphaproteobacteria, 6.3\%), otu12 (Achromobacter, 4.2\%), otu14 (a member of the Enterobacteriaceae, 2.5\%), otu15 (Propionibacterium, 1.9\%), otu16 (Pseudomonas, 2.3\%), otu18 (Stenotrophomonas, 1.3\%), otu19 (Undibacterium, 1.4\%), and otu20 (a member of the Xanthomonadaceae, 1.2\%). The otu21 (Acinetobacter) had relative abundance of less than $1 \%$ in both 
Hfg2 and Hfg3 samples. The OTUs otu08, otu09, otu10, otu12, otu16, otu20, otu23, otu24, otu25, and otu26, were detected only in the Hfg3 sample.

In summary, pyrosequencing revealed Pseudomonas as the most abundant genus in the gut of all Hessian fly larval instars. Pseudomonas corresponding to otu01 exhibited the highest frequency, but its relative abundance decreases as larvae developed into later instars. On the other hand, Pseudomonas bacteria corresponding to otu06 increased in abundance from less than $1 \%$ in the gut of 1 st and 2 nd instars to more than $13 \%$ in the gut of 3rd instar. In addition to Pseudomonas, Buttiauxella was the second most abundant bacteria in the 2nd instar, and remained relatively high abundance in the 3rd instar. Among the three larval instars, the gut of the 3rd instars exhibited the highest number of OTUs and highest richness estimates in spite of having fewer sequences, indicating that the third instar larvae contained more diverse microbes in the gut. At the genus level, the gut of 1 st instar contained only two different types of bacteria that exceeded $1 \%$ in relative abundance, the gut of 2 nd instar contained three different types of bacteria that exceeded $1 \%$ in abundance, but the gut of $3 \mathrm{rd}$ instar contained 13 different types of bacteria that exceeded $1 \%$ in abundance. The biological significance of increased diversity in bacterial composition in 3rd instar larvae remain to be studied.

As Pseudomonas was found to be the most dominant bacteria genera in the gut of Hessian fly larvae, we determined its population dynamics during insect's various developmental stages including pupae and adults. Measurement of population levels is a step toward its functional characterization as the dynamics curve of a bacterium emphasizes its importance in a particular developmental stage of the insect host. To this end, the relative abundance of Pseudomonas 16S rDNA in Hessian fly's developmental stages is shown in Figure 4. In the first instar larvae, peak level of 16S rDNA was observed on day 0 (in biotype GP) or day 1 (in field populations from Grayson, TX and Fannin, TX, USA); but it fell sharply thereafter during the same instar stage. However during the later developmental stages, high levels of 16S rDNA were observed consistently except in biotype GP adults (females). Higher abundance of Pseudomonas in freshly hatched or 1 day old larvae of Hessian fly assumes significance as the early first instar larvae of Hessian fly (and other gall midges) is a critical stage that determines the compatibility of the interaction with the wheat (host) plant [26,27]. As opposed to laboratory reared populations, the field populations of Hessian fly feed on diverse wheat cultivars and are continuously exposed to a variety of biotic and abiotic stresses. As a result, field populations experience selection pressures due to different nutritive qualities of wheat varieties, various management practices (such as use of insecticides and cultivation of resistant wheat), natural enemies (pathogens, predators and parasitoids), and other environmental conditions which ultimately can alter the frequency of gut microbes. It is possible that because of the benefits imparted by Pseudomonas to adult flies in the field, this bacterium is maintained at higher frequency compared to that in laboratory adults (Figure 4). Thus, it was perhaps not surprising to see different titers of Pseudomonas in adults of laboratory (biotype GP) and field (Grayson and Fannin) populations. 
Figure 4. Pseudomonas 16S rDNA levels in Hessian fly developmental stages.

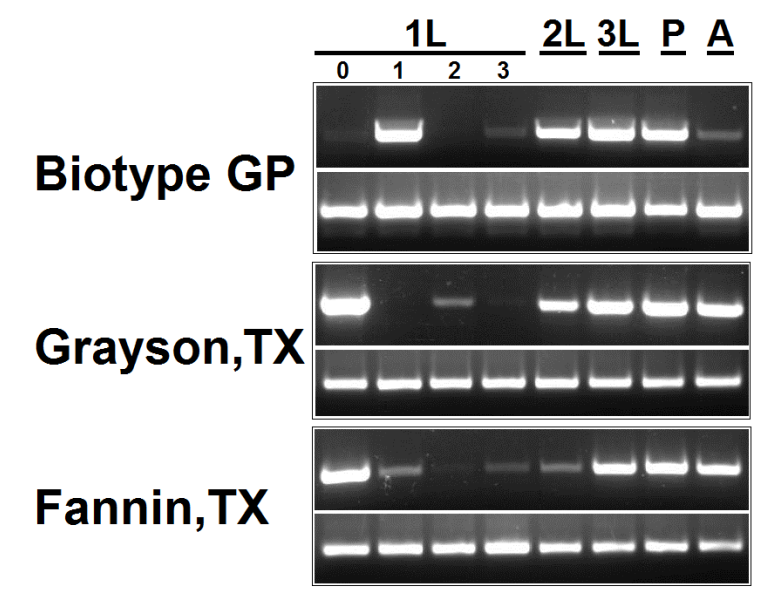

Results of semi-quantitative PCR in different developmental stages of biotype GP and of two field populations (Grayson, TX, USA, and Fannin, TX, USA) are shown. For each panel, upper gel is for Pseudomonas (33 cycles; 401 bp product) and lower gel is for Hessian fly actin gene (30 cycles: 740 bp product). Figures on the top refer to various developmental stages i.e., 1L: first instar larvae; 2L: second instar larvae (8 days old); 3L: third instar larvae (14 days old); P: pupae; and A: adults. In first instar larval stage, Pseudomonas $16 \mathrm{~S}$ rDNA levels were estimated at four different time points i.e., 0 : freshly hatched; 1 : one day old; 2: two days old; and 3: three days old.

Earlier, Pseudomonas has been found in gut of dipteran flies and other insects [28-34]; however, the definite evidence on its role in insect biology is lacking. The Pseudomonas from olive fly gut hydrolyzes the proteins of olive flesh, suggesting that the olive fly is dependent upon its symbiont for the utilization of its plant [35]. Hessian fly is a member of the gall midges. Galling insects induce the formation of various types of galls [8], a process that requires reprogramming of biochemical and physiological pathways of host plants. The fact that similar bacteria exist in Hessian fly-infested wheat indicates that Hessian fly larvae transmit symbiotic bacteria into host plant tissue during feeding [15]. These results suggest that microbes associated with Hessian fly may play roles both in the physiological process of the insect development and in the interaction with host plants. So far, Hessian fly is the only gall midge in which bacterial association is reported [12-15]. This research is the first to systematically analyze the microbial community in a galling insect. The discovery of diverse microbes in the gut of Hessian fly larvae should provide a foundation for future research to characterize their functions, and to stimulate similar studies on other galling insects.

\section{Experimental Section}

\subsection{Insect and Infestation}

Larval samples used for pyrosequencing were the progeny of a fly colony that originated from a field infestation collected in Kansas [7]. The majority (99\%) of the insects in colony were biotype GP (the Great Plains) although biotypes A, B and others were also found in low frequencies [36]. The susceptible wheat cultivar Karl 92 was used for insect rearing. Plants were grown in environmental chambers at $20{ }^{\circ} \mathrm{C}$ with a 12:12 (L:D) photoperiod. Seedlings at 1.5 leaf-stage (full grown first leaf with second leaf just emerged) were infested with Hessian fly eggs by confining adult flies close to 
plants with a cage with mesh screen. Average egg density was approximately 8 per plant, resulting in approximately 6 larvae per plant.

To estimate population dynamics of Pseudomonas, various developmental stages of Hessian fly were collected from first generation of populations which were established from samples collected in Grayson and Fannin counties, TX, where large infestations were present [37]. Populations from field collections were established by following the protocol as described above.

\subsection{Gut Tissue and DNA Preparation}

Hessian larval gut consists of a very small foregut, a major midgut, and a very small hindgut [38]. Gut tissues (containing all the three parts of the gut) were obtained from first (1-3 days old), second (6-8 days old), and third (13-15 days old) instar larvae, respectively. Two hundred guts each from first and second instars, and 100 guts from third instars, were prepared by dissecting larvae under a dissecting microscope. The dissected guts were immediately put into TE buffer ( $\mathrm{pH} 7.5)$ and homogenized by using a pellet pestle and electric drill for about $20 \mathrm{~s} /$ sample. Genomic DNA was extracted by Cetyl trimethylammonium bromide (CTAB) DNA extraction method [39]. Briefly, $250 \mu \mathrm{L}$ of tissue homogenates were incubated with $500 \mu \mathrm{L}$ of $2 \times$ CTAB buffer $(100 \mathrm{mM}$ Tris-HCl, $\mathrm{pH} 8,1.4 \mathrm{M} \mathrm{NaCl}, 20 \mathrm{mM}$ EDTA, 2\% (w/v) CTAB and 0.2\% $(v / v) \beta$-mercaptoethanol) at $65{ }^{\circ} \mathrm{C}$ for $30 \mathrm{~min}$. The DNA was then extracted using the phenol-chloroform method as described by [40] and precipitated using isopropanol. The DNA pellet was resuspended in $50 \mu \mathrm{L}$ of nuclease-free water and quantified using a NanoDrop-1000 spectrophotometer (Thermo Scientific, Wilmington, DE, USA).

Three reverse PCR primers, U529R-FC-A33, U529R-FC-A40, U529R-FC-A90 were synthesized for amplifying V3 region of microbial 16S rRNA genes. Each reverse primer consisted of an added sequence for sequencing (primer A, italic), a unique barcode (underlined), and a 16S-specific sequence (bold). Each reverse primer was used for PCR for a different sample, namely Hfg1, Hfg2, or Hfg3. Hfg1, Hfg2, and Hfg3 were derived from the gut of the first, second, and third instar larvae, respectively. The sole forward PCR primer, U341F-FC-B, consisted of an added sequence (primer B) and a 16S-specific sequence (bold).

\subsection{Template Preparation and Pyrosequencing}

To construct bacterial libraries for pyrosequencing, the forward primer U341F-FC-B and one of the three reverse primers (Table 2) were used to amplify the V3 variable region of the 16S rRNA gene from different DNA samples. Specifically, the primer with the barcode TGATG was used to amplify gut DNA from the first instar larvae (Hfg1), the primer with the barcode TCACT was used to amplify gut DNA from the second instar larvae (Hfg2), whereas the primer with the barcode ATACG was used to amply gut DNA from the third instar larvae (Hfg3) as described previously [41]. The samples were amplified in a $25 \mu \mathrm{L}$ mixture containing $1 \mu \mathrm{L}(10 \mathrm{ng} / \mu \mathrm{L})$ of DNA as template, $12.5 \mu \mathrm{L}$ of $2 \times$ PCR master mix from Promega (with a final concentration of $0.4 \mathrm{mM}$ each deoxynucleoside triphosphate, $1.5 \mathrm{mM} \mathrm{MgCl} 2$ and 0.625 units of Taq DNA polymerase in PCR reaction buffer $\mathrm{pH} 8.5$ ), and $0.32 \mathrm{mM}$ $(1 \mu \mathrm{L})$ each primer. The reactions were performed on a PTC100 Thermal Cycler (MJ Research, Watertown, MA, USA). The reaction cycle included an initial denaturation at $95{ }^{\circ} \mathrm{C}$ for $5 \mathrm{~min}$, followed by 30 cycles of $30 \mathrm{~s}$ each at 95,55 , and $72{ }^{\circ} \mathrm{C}$, with a final extension of $5 \mathrm{~min}$ at $72{ }^{\circ} \mathrm{C}$. After 
amplification, the PCR products were purified using a Qiagen QIAquick PCR purification kit (Qiagen, Valencia, CA, USA) and were quantified using a NanoDrop-1000 spectrophotometer (Thermo Scientific, Wilmington, DE, USA). Equal amounts of the PCR products from different samples were pooled and sequenced from the reverse direction by the 454 Life Sciences Company (Branford, CT, USA).

Table 2. Primers for template preparation and pyrosequencing.

\begin{tabular}{ccc}
\hline Primer Name & Primer Sequence & Targeted Sample \\
\hline U529R-FC-A33 & 5'-GCCTCCCTCGCGCCATCAGTGATGACCGCGGCKGCTGGC & Hfg1 \\
U529R-FC-A40 & 5'-GCCTCCCTCGCGCCATCAG $\underline{\text { TCACTACCGCGGCKGCTGGC }}$ & Hfg2 \\
U529R-FC-A90 & 5'-GCCTCCCTCGCGCCATCAGATACGACCGCGGCKGCTGGC & Hfg3 \\
Primer A & 5'-GCCTCCCTCGCGCCATCAG & \\
U529R & 5'-ACCGCGGCKGCTGGC & \\
U341F-FC-B & 5'-GCCTTGCCAGCCCGCTCAGCCTACGGGRSGCAGCAG \\
Primer B & 5'-GCCTTGCCAGCCCGCTCAG \\
U341F & 5'-CCTACGGGRSGCAGCAG & \\
\hline
\end{tabular}

\subsection{Sequence Processing and Analysis}

Based on the 5'-barcode sequences, raw pyrosequencing data was assigned to each sample and 454 sequencing adapters were removed. Reads were discarded if they were $<90$ bases or $>135$ bases, or with one or more undetermined or ambiguous nucleotides, or with no valid barcode. After that, sequences were analyzed using Mothur software [22], based on the Schloss SOP [42]. Reads were aligned to the ARB-Silva database of $16 \mathrm{~S}$ rRNA sequences [43,44]. Pre-clustering was performed (clustering by $2 / 100$ base difference) to reduce pyrosequencing noise i.e., the potential sequence variation introduced due to sequencing error at the nucleotide level [45]. Chimeric sequences were identified and removed using Perseus which takes into account the relative abundance [46]. Remaining reads were assigned into OTUs at different similarity cutoffs ( $\geq 90 \%, \geq 95 \%, \geq 97 \%, \geq 99 \%$ ) using "average neighbor" algorithm. OTUs (assigned at $\geq 97 \%$ similarity cutoff) encompassing only single read were not analyzed further. Reads in each OTU (assigned at $\geq 97 \%$ similarity cutoff) were classified into various genera (or identifiable taxon) using the Bayesian method (bootstrap limit 80\%) [47]. The ARB-Silva database was used as reference for taxonomic classification. Additionally, SINA (SILVA Incremental Aligner) tool was used (employing the "least common ancestor" method) to support taxonomic inferences of "ARB-Silva database" search for OTUs identified as "eukaryota" or "unknown" at kingdom level [48]. The sequence reads matching to cyanobacteria-chloroplast, Magnoliophyta, and Insecta groups were further identified through wheat and Hessian fly references using NCBI's blastn tool. Representative sequence for each of the top 20 OTUs (accounting for 94.34\% reads classified to "bacteria" kingdom) was used to deduce the phylogenetic inference in MEGA5.05 [24]. The phylogeny was inferred using the Maximum Likelihood method based on the Tamura-Nei model [23]. The bootstrap test with 1000 replicates was conducted to test the reliability [25]. Initial tree for the heuristic search was obtained by applying the Neighbor-Joining method to a matrix of pairwise distances estimated using the Maximum Composite Likelihood approach. The number of observed OTUs was used as a measure of community richness in the gut of larval instars. Estimates of two non-parametric measures of richness, Ace [49] and Chao [50], were performed in Mothur [22]. 
Rarefaction analysis and coverage estimation were also performed in Mothur [22]. All sequence data were deposited in the Sequence Read Archive, NCBI under BioProject ID\# PRJNA231475 (SRP033878).

\subsection{Estimation of Pseudomonas 16S rDNA Level in Hessian Fly Developmental Stages}

DNA extractions from various developmental stage samples of biotype GP, Grayson, TX and Fannin, TX populations were performed as described above. The Pseudomonas-specific primers (forward: TAGAGAGRWGCWYGCTTCTCTTGA and reverse: CAATTACGTATTAGGTAACTGCCC were used. Hessian fly actin gene (accession no. AF017427; forward: ATGTGTGACGACGAAGTTGCT and reverse: GGCAACATACATGGCTGGTG-3') was used as a control for template quality and quantity [51]. The samples were amplified in a $25 \mu \mathrm{L}$ mixture containing $1 \mu \mathrm{L}(10 \mathrm{ng} / \mu \mathrm{L})$ template DNA, $12.5 \mu \mathrm{L} 2 \times$ PCR master mix from Promega (with a final concentration of $0.4 \mathrm{mM}$ each deoxynucleoside triphosphate, $1.5 \mathrm{mM} \mathrm{MgCl}_{2}$ and 0.625 units of Taq DNA polymerase in PCR reaction buffer $\mathrm{pH} 8.5$ ) and $0.32 \mathrm{mM}$ each primer. The reactions were performed on a PTC100 Thermal Cycler (MJ Research, Watertown, MA, USA) and the reaction cycle included an initial denaturation of $5 \mathrm{~min}$ at $95{ }^{\circ} \mathrm{C}$ followed by variable numbers of cycles of $30 \mathrm{~s}$ at $94{ }^{\circ} \mathrm{C}, 30 \mathrm{~s}$ at $55{ }^{\circ} \mathrm{C}$, and $30 \mathrm{~s}$ at $72{ }^{\circ} \mathrm{C}$, with a final extension of $5 \mathrm{~min}$ at $72{ }^{\circ} \mathrm{C}$.

\section{Acknowledgments}

The authors thank Kun Yan Zhu from the Department of Entomology, Kansas State University, Manhattan, KS, and Guihua Bai from the Hard Winter Wheat Genetics Research Unit, USDA-ARS at Manhattan, KS, for reviewing an earlier version of the manuscript. This paper is contribution 11-123-J from the Kansas Agricultural Experiment Station. Mention of commercial or proprietary product does not constitute endorsement by USDA.

\section{Author Contributions}

RB, SHH, MSC conceived and designed the experiments; SHH, JCR, RJW, JJS, MSC contributed reagents and materials; RB performed the experiments and analyzed the data; RB, SHH, JCR, RJW, JJS, MSC wrote the manuscript.

\section{Conflicts of Interest}

The authors declare no conflict of interest.

\section{References}

1. Dillon, R.; Dillon, V. The gut bacteria of insects: nonpathogenic interactions. Annual Reviews in Entomology 2004, 49, 71-92.

2. Engel, P.; Moran, N.A. The gut microbiota of insects-diversity in structure and function. FEMS Microbiol. Rev. 2013, 37, 699-735.

3. Holmes, E.; Nicholson, J. Variation in gut microbiota strogly influences individual rodent phenotypes. Toxicological Sciences 2005, 87, 1-2. 
4. Chapman, R.F. The Insects: Structure And Function; Cambridge University press: Cambridge, UK, 1998.

5. Buchner, P. Endosymbiosis of Animals with Plant Microorganims; John Wiley \& Sons: Chichester, UK, 1965.

6. Hansen, A.K.; Moran, N.A. The impact of microbial symbionts on host plant utilization by herbivorous insects. Mol. Ecol. 2013, 23, 1473-1496.

7. Gagne, R.; Hatchett, J. Instars of the Hessian fly (Diptera: Cecidomyiidae). Ann. Entomol. Soc. Am. 1989, 82, 73-79.

8. Ananthakrishnan, T.N. Biology of gall insects; Oxford and IBH: New Delhi, India, 1984.

9. Pauly, P.J. Fighting the Hessian fly: American and British responses to insect invasion 17761789. Environ. Hist. 2002, 7, 485-507.

10. Harris, M.; Stuart, J.; Mohan, M.; Nair, S.; Lamb, R.; Rohfritsch, O. Grasses and gall midges: plant defense and insect adaptation. Annu. Rev. Entomol. 2003, 48, 549-577.

11. Harris, M.; Freeman, T.; Rohfritsch, O.; Anderson, K.; Payne, S.; Moore, J. Virulent Hessian fly (Diptera: Cecidomyiidae) larvae induce a nutritive tissue during compatible interactions with wheat. Ann. Entomol. Soc. Am. 2006, 99, 305-316.

12. Boosalis, G. Hessian fly in relation to the development of crown and basal stem rot of wheat. Phytopathology 1954, 44, 224-229.

13. Mittapalli, O.; Shukle, R.H.; Sardesai, N.; Giovanini, M.P.; Williams, C.E. Expression patterns of antibacterial genes in the Hessian fly. J. Insect Physiol. 2006, 52, 1143-1152.

14. Bansal, R. Hessian Fly Associated Microbes: Dynamics, Transmission And Essentiality; ProQuest, UMI Dissertation Publishing: Ann Arbor, MI, USA, 2010.

15. Bansal, R.; Hulbert, S.; Schemerhorn, B.; Reese, J.C.; Whitworth, R.J.; Stuart, J.J.; Chen, M. Hessian fly-associated bacteria: transmission, essentiality, and composition. PloS One 2011, 6, e23170.

16. Warnecke, F.; Luginbühl, P.; Ivanova, N.; Ghassemian, M.; Richardson, T.H.; Stege, J.T.; Cayouette, M.; McHardy, A.C.; Djordjevic, G.; Aboushadi, N. Metagenomic and functional analysis of hindgut microbiota of a wood-feeding higher termite. Nature 2007, 450, 560-565.

17. Cox-Foster, D.L.; Conlan, S.; Holmes, E.C.; Palacios, G.; Evans, J.D.; Moran, N.A.; Quan, P.; Briese, T.; Hornig, M.; Geiser, D.M. A metagenomic survey of microbes in honey bee colony collapse disorder. Science 2007, 318, 283-287.

18. Moran, N.A.; Hansen, A.K.; Powell, J.E.; Sabree, Z.L. Distinctive gut microbiota of honey bees assessed using deep sampling from individual worker bees. PLoS One 2012, 7, e36393.

19. Neefs, J.; Van de Peer, Y.; De Rijk, P.; Goris, A.; De Wachter, R. Compilation of small ribosomal subunit RNA sequences. Nucleic Acids Res. 1991, 19, 1987-2015.

20. Fox, G.E.; Stackebrandt, E.; Hespell, R.B.; Gibson, J.; Maniloff, J.; Dyer, T.A.; Wolfe, R.S.; Balch, W.E.; Tanner, R.S.; Magrum, L.J.; et al. The phylogeny of prokaryotes. Science 1980, 209, 457-463.

21. Huse, S.M.; Dethlefsen, L.; Huber, J.A.; Welch, D.M.; Relman, D.A.; Sogin, M.L. Exploring microbial diversity and taxonomy using SSU rRNA hypervariable tag sequencing. PLoS genetics 2008, 4, e1000255. 
22. Schloss, P.D.; Westcott, S.L.; Ryabin, T.; Hall, J.R.; Hartmann, M.; Hollister, E.B.; Lesniewski, R.A.; Oakley, B.B.; Parks, D.H.; Robinson, C.J. Introducing mothur: open-source, platformindependent, community-supported software for describing and comparing microbial communities. Appl. Environ. Microbiol. 2009, 75, 7537-7541.

23. Tamura, K.; Nei, M. Estimation of the number of nucleotide substitutions in the control region of mitochondrial DNA in humans and chimpanzees. Mol. Biol. Evol. 1993, 10, 512-526.

24. Tamura, K.; Peterson, D.; Peterson, N.; Stecher, G.; Nei, M.; Kumar, S. MEGA5: molecular evolutionary genetics analysis using maximum likelihood, evolutionary distance, and maximum parsimony methods. Mol. Biol. Evol. 2011, 28, 2731-2739.

25. Felsenstein, J. Confidence limits on phylogenies: an approach using the bootstrap. Evolution 1985, 39, 783-791.

26. Byers, R.A.; Gallun, R.L. Ability of the Hessian fly to stunt winter wheat: effect of larval feeding on elongation of leaves. J. Econ. Entomol. 1972, 65, 955-958.

27. Rohfritsch, O. Patterns in gall development. In Biology of insect-induced galls; Shorthouse, J.D., Rohfritsch, O., Eds.; Oxford University Press: Oxford, UK, 1992; pp. 60-86.

28. Prabhakar, C.S.; Sood, P.; Kanwar, S.S.; Sharma, P.N.; Kumar, A.; Mehta, P.K. Isolation and characterization of gut bacteria of fruit fly, Bactrocera tau (Walker). Phytoparasitica 2013, 41, 193-201.

29. Jing, X.; Wong, A.C.; Chaston, J.M.; Colvin, J.; McKenzie, C.L.; Douglas, A.E. The bacterial communities in plant phloem-sap-feeding insects. Mol. Ecol. 2014, 23, 1433-1444.

30. Wei, T.; Ishida, R.; Miyanaga, K.; Tanji, Y. Seasonal variations in bacterial communities and antibiotic-resistant strains associated with green bottle flies (Diptera: Calliphoridae). Appl. Microbiol. Biotechnol. 2014, 98, 4197-4208.

31. Broderick, N.A.; Robinson, C.J.; McMahon, M.D.; Holt, J.; Handelsman, J.; Raffa, K.F. Contributions of gut bacteria to Bacillus thuringiensis-induced mortality vary across a range of Lepidoptera. BMC Biology 2009, 7, 11.

32. Stavrinides, J.; McCloskey, J.K.; Ochman, H. Pea aphid as both host and vector for the phytopathogenic bacterium Pseudomonas syringae. Appl. Environ. Microbiol. 2009, 75, 2230-2235.

33. Zhang, F.; Huang, Y.H.; Liu, S.Z.; Zhang, L.; Li, B.T.; Zhao, X.X.; Fu, Y.; Liu, J.J.; Zhang, X.X. Pseudomonas reactans, a bacterial strain isolated from the intestinal flora of Blattella germanica with anti-Beauveria bassiana activity. Environ. Entomol. 2013, 42, 453-459.

34. Morales-Jiménez, J.; de León, A.V.; García-Domínguez, A.; Martínez-Romero, E.; Zúñiga, G.; Hernández-Rodríguez, C. Nitrogen-fixing and uricolytic bacteria associated with the gut of Dendroctonus rhizophagus and Dendroctonus valens (Curculionidae: Scolytinae). Microb. Ecol. 2013, 66, 200-210.

35. Hagen, K.S. Dependence of the olive fly, Dacus oleae, larvae on symbiosis with Pseudomonas savastanoi for the utilization of olive. 1966, 209, 423-424.

36. Harris, M.; Rose, S. Temporal changes in the egglaying behaviour of the Hessian fly. Entomol. Exp. Appl. 1989, 53, 17-29.

37. Chen, M.; Echegaray, E.; Whitworth, R.J.; Wang, H.; Sloderbeck, P.E.; Knutson, A.; Giles, K.L.; Royer, T.A. Virulence analysis of Hessian fly populations from Texas, Oklahoma, and Kansas. J. Econ. Entomol. 2009, 102, 774-780. 
38. Stuart, J.; Hatchett, J. Morphogenesis and cytology of the salivary gland of the Hessian fly, Mayetiola destructor (Diptera: Cecidomyiidae). Ann. Entomol. Soc. Am. 1987, 80, 475-482.

39. Doyle, J.J. A rapid DNA isolation procedure for small quantities of fresh leaf tissue. Phytochem. Bull 1987, 19, 11-15.

40. Sambrook, J.; Fritsch, E.F.; Maniatis, T. Molecular cloning; Cold spring harbor laboratory press: New York, NY, USA, 1989; Volume 2.

41. Bansal, R.; Mian, M.; Michel, A.P. Microbiome diversity of Aphis glycines with extensive superinfection in native and invasive populations. Env. Microbiol. Rep. 2014, 6, 57-69.

42. Schloss, P.D.; Gevers, D.; Westcott, S.L. Reducing the effects of PCR amplification and sequencing artifacts on 16S rRNA-based studies. PloS One 2011, 6, e27310.

43. Pruesse, E.; Quast, C.; Knittel, K.; Fuchs, B.M.; Ludwig, W.; Peplies, J.; Glöckner, F.O. SILVA: a comprehensive online resource for quality checked and aligned ribosomal RNA sequence data compatible with ARB. Nucleic Acids Res. 2007, 35, 7188-7196.

44. Pruesse, E.; Peplies, J.; Glöckner, F.O. SINA: accurate high-throughput multiple sequence alignment of ribosomal RNA genes. Bioinformatics 2012, 28, 1823-1829.

45. Huse, S.M.; Welch, D.M.; Morrison, H.G.; Sogin, M.L. Ironing out the wrinkles in the rare biosphere through improved OTU clustering. Environ. Microbiol. 2010, 12, 1889-1898.

46. Quince, C.; Lanzen, A.; Davenport, R.J.; Turnbaugh, P.J. Removing noise from pyrosequenced amplicons. BMC Bioinformatics 2011, 12, 38.

47. Wang, Q.; Garrity, G.M.; Tiedje, J.M.; Cole, J.R. Naive Bayesian classifier for rapid assignment of rRNA sequences into the new bacterial taxonomy. Appl. Environ. Microbiol. 2007, 73, 5261-5267.

48. Pruesse, E.; Peplies, J.; Glöckner, F.O. SINA: accurate high-throughput multiple sequence alignment of ribosomal RNA genes. Bioinformatics 2012, 28, 1823-1829.

49. Chao, A.; Lee, S. Estimating the number of classes via sample coverage. Journal of the American Statistical Association 1992, 87, 210-217.

50. Chao, A. Nonparametric estimation of the number of classes in a population. Scand. J. Stat. 1984, 265-270.

51. Giovanini, M.P.; Puthoff, D.P.; Nemacheck, J.A.; Mittapalli, O.; Saltzmann, K.D.; Ohm, H.W.; Shukle, R.H.; Williams, C.E. Gene-for-gene defense of wheat against the Hessian fly lacks a classical oxidative burst. Mol. Plant Microbe Interact. 2006, 19, 1023-1033.

(C) 2014 by the authors; licensee MDPI, Basel, Switzerland. This article is an open access article distributed under the terms and conditions of the Creative Commons Attribution license (http://creativecommons.org/licenses/by/3.0/). 